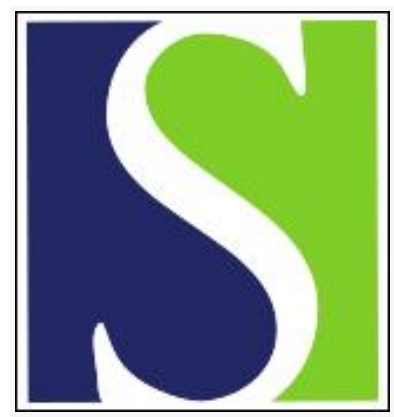

Scand J Work Environ Health 2020;46(1):50-59

https://doi.org/10.5271/sjweh.3837

Published online: 06 Jun 2019, Issue date: 01 Jan 2020

Individual placement and support for young adults at risk of early work disability (the SEED trial). A randomized controlled trial

by Sveinsdottir V, Lie SA, Bond GR, Eriksen HR, Tveito TH, Grasdal AL, Reme SE

A randomized controlled trial was conducted of individual placement and support (IPS) for young adults with various social or health-related problems at risk of work disability. The odds of gaining competitive employment were $10.39(95 \% \mathrm{Cl} 2.79-38.68)$ times higher for IPS than traditional vocational rehabilitation ( $48 \%$ versus $8 \%)$. IPS shows promise for early intervention diverting young adults from disability to employment.

Affiliation: NORCE Norwegian Research Centre, POB 7810, 5020 Bergen, Norway. visv@norceresearch.no

Refers to the following text of the Journal: 2019;45(1):33-41

The following article refers to this text: 2020;46(4):410-416

Key terms: early work disability; health; health; individual placement and support; intervention; NEET; randomized controlled trial; RCT; SEED trial; supported employment; vocational rehabilitation; work; work; work; work disability; young adult

This article in PubMed: www.ncbi.nlm.nih.gov/pubmed/31170299

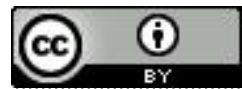




\title{
Individual placement and support for young adults at risk of early work disability (the SEED trial). A randomized controlled trial
}

\author{
by Vigdis Sveinsdottir, MSc, ${ }^{1}$ Stein Atle Lie, PhD, ${ }^{2}$ Gary R Bond, PhD, ${ }^{3}$ Hege R Eriksen, PhD, ${ }^{4}$ Torill H Tveito, PhD, ${ }^{1,5}$ \\ Astrid L Grasdal, DrPol, ${ }^{6}$ Silje E Reme, PhD ${ }^{7}$
}

\begin{abstract}
Sveinsdottir V, Lie SA, Bond GR, Eriksen HR, Tveito TH, Grasdal AL, Reme SE. Individual placement and support for young adults at risk of early work disability (the SEED trial). A randomized controlled trial. Scand J Work Environ Health. 2020;46(1):50-59. doi:10.5271/sjweh.3837
\end{abstract}

\begin{abstract}
Objectives Individual placement and support (IPS) is an effective approach for helping people with severe mental illness gain employment. This study aimed to investigate if IPS can be effectively repurposed to support young adults at risk of early work disability due to various social and health related problems.
\end{abstract}

Methods A randomized controlled trial including 96 young adults (18-29 years; $68 \%$ men) was conducted in Norway. Participants were not in employment, education, or training, received temporary benefits due to social or health-related problems, and were eligible for traditional vocational rehabilitation (TVR). Participants were randomized to IPS ( $\mathrm{N}=50)$ or TVR $(\mathrm{N}=46)$. Self-reported data were collected at baseline and at 6- and 12-months follow-up. The primary outcome was obtaining any paid employment in the competitive labor market during followup. Secondary outcomes were physical and mental health, well-being, coping, alcohol consumption, and drug use.

Results Significantly more IPS participants obtained competitive employment compared to TVR participants during 12 -months follow-up ( $48 \%$ versus $8 \%$; odds ratio $10.39,95 \%$ confidence interval $2.79-38.68$ ). The IPS group reported significantly better outcomes than the TVR group in subjective health complaints, helplessness, and hopelessness. In post hoc analyses adjusted for baseline and missing data, the IPS group reported significantly better outcomes on these measures in addition to level of disability, optimism about future well-being, and drug use.

Conclusions IPS is effective for young adults at risk of early work disability. IPS was superior to TVR in increasing competitive employment and promoted improvements in some non-vocational outcomes. IPS services should be offered to improve employment rates in this vulnerable group.

Key terms health; intervention; NEET; RCT; supported employment; vocational rehabilitation; work.

High rates of young people who are not in employment, education, or training (NEET) represent an important international challenge (1). The NEET population is diverse, and includes individuals who are short-term unemployed or in temporary transition-phases, as well as other more vulnerable groups at higher risk of lifelong disengagement (2).

Exclusion from the labor market is associated with adverse health effects $(3,4)$ and leads to considerable societal costs (1). In Norway, disability benefits are offered as income compensation for individuals with permanently reduced earning capacity. During the last decade, there has been a shift in disability benefits toward younger recipients, and the share of young adults aged 18-29 has increased considerably (5). This group differs from the older beneficiaries as the majority (56\%) of young recipients are male compared to $42 \%$ across all age groups. In addition, $63 \%$ of the disability determinations in the 18-29-year-old age group are attributed to mental and behavioral disorders compared to $35 \%$ across

1 NORCE Norwegian Research Centre, Bergen, Norway

2 Department of Clinical Dentistry, Faculty of Medicine, University of Bergen, Bergen, Norway

3 Westat, Lebanon, NH, USA

4 Department of Sport, Food and Natural Sciences, Western Norway University of Applied Sciences, Bergen, Norway

5 Department of Health, Social and Welfare Studies, University of South-Eastern Norway, Horten, Norway

6 Department of Economics, University of Bergen, Bergen, Norway

7 Department of Psychology, Faculty of Social Sciences, University of Oslo, Oslo, Norway

Correspondence to: Vigdis Sveinsdottir, NORCE Norwegian Research Centre, POB 7810, 5020 Bergen, Norway. [E-mail: visv@norceresearch.no] 
all age groups. The dominant role of mental and behavioral disorders is not unique in a Norwegian context but is among the leading causes for years lost due to disability among youth in most high-income countries (6).

Given the heterogeneity of the NEET population, efforts to integrate young people into employment should target specific subgroups. Existing policies bear the risk of being more appropriate for those who are work-ready while failing to reach more disadvantaged groups (1). A recent systematic review found limited evidence for effective re-engagement interventions for NEET, and emphasized that existing knowledge is insufficient to guide policy-makers in the planning and implementation of new programs (7).

The individual placement and support (IPS) model of supported employment is an evidence-based intervention that is effective in improving competitive employment outcomes for patients with severe mental illness (8). While IPS generally does not directly improve non-vocational outcomes (9), competitive employment has beneficial effects, including reduced symptoms and increased self-esteem (10). The IPS model is based on eight principles emphasizing focus on competitive employment, rapid job search, no exclusion due to evaluation of work readiness or other reasons, attention to client preferences, long-term individualized support, integrated services, systematic job development, and benefits counseling (11). While the model was originally developed for patients with severe mental illness, recent studies suggest that it may be effective for other disability groups (12). No previous studies have however investigated the effectiveness of IPS for young adults at risk of early work disability due to various social and health-related problems, that may or may not involve mental illness.

The project "Supported Employment and preventing Early Disability" (SEED) aimed to investigate whether IPS can be repurposed to serve NEET at risk of early work disability in Norway. The SEED trial also aimed to evaluate the effectiveness of IPS compared to traditional vocational rehabilitation (TVR) on outcomes of competitive employment as well as physical and mental health and well-being.

\section{Methods}

\section{Trial design}

SEED was a two-armed randomized controlled trial (RCT) comparing IPS to TVR. The trial was investigator-initiated and funded by the Research Council of Norway. The Norwegian Regional Committees for Medical and Health Research Ethics exempted the project as it did not fall under the Health Research Act
(13) and referred it to the Data Protection Services at the Norwegian Centre for Research Data, which approved the project (project \#38271). All participants gave written informed consent before study inclusion, and the ethical principles of the Helsinki declaration were followed. The project was registered in ClinicalTrials.gov (registration \#NCT02375074), and the study protocol is available online (14).

\section{Eligibility criteria}

The eligibility criteria for SEED included: (i) age 18-29 at year of inclusion; (ii) not in employment or education; (iii) receiving temporary benefits from the Norwegian Labor and Welfare Service (NAV), and thereby required to keep up to an activity plan involving treatment and/ or vocational rehabilitation while work ability is being assessed; (iv) considered eligible for and expected to participate in the TVR intervention "traineeship in a sheltered business" by the individual's caseworker at NAV. Eligibility applies to individuals with impaired work capability that require close and broad supervision and assistance (15).

Exclusion criteria were not expressing interest in getting help to obtain competitive work upon inclusion and insufficient language skills to answer questionnaires in Norwegian. There were no exclusion criteria based on diagnosis, and individuals with any type of social or health-related problem were invited to participate.

\section{Recruitment, randomization, and blinding}

The recruitment period lasted from June 2014 through December 2016. Eligible participants were referred to information meetings by staff at one central and nine local labor and welfare offices in and around the city of Bergen, or at a secondary care district psychiatric center with subsequent follow-up at the local labor and welfare office. Eligible participants were given verbal and written information by the project coordinator and invited to participate.

After participants agreed to participate and completed the baseline questionnaire, they were randomly assigned to one of two conditions using a computergenerated randomization sequence with a block size of 8 and a 1:1 randomization ratio to the two groups. The ratio was temporarily changed for a period of three months to 2:1 (with two participants assigned to IPS for every one assigned to TVR), in order to enable sufficient caseloads for the job specialists. Staff at Uni Research Health carried out the randomization and communicated the results to the individual's caseworker at NAV by email. Created by a statistician who had no contact with the participants, the randomization sequence was concealed from participants, service 
providers, and the researcher responsible for controlling the data analyses. The researcher responsible for quality control of the data analyses was blinded for intervention assignment.

\section{Interventions}

Both IPS and TVR were offered by vocational rehabilitation organizations overseen by the NAV, which provides employment services to temporary benefit recipients in Norway.

Individual placement and support (IPS). IPS participants were referred to an organization with two trained job specialists. The specialists sought to follow the IPS principles (11) and find a good job match while avoiding the use of prevocational training or subsidized or unpaid work. Unlike traditional vocational approaches, IPS focuses exclusively on competitive employment, clients are not screened for job readiness, client preferences guide choices and decisions, and job specialists continue to provide ongoing support after clients attain employment. An IPS team leader supervised the job specialists, and an external IPS trainer advised the team. Because the study population had various social and healthrelated challenges that did not necessarily involve mental illness, the IPS principle of integrating services with mental health treatment was not implemented, although job specialists contacted health personnel involved in the treatment of individual participants in cases where this was applicable and accepted by the participant. The intervention was offered for up to three years, and the duration and intensity depended on individual needs and preferences.

Traditional vocational rehabilitation (TVR). The TVR group was referred to an organization offering a traditional employment scheme called "traineeship in a sheltered business", aiming to improve the opportunities for finding a job (15). This intervention represented treatment as usual and served as an active control condition. The traineeships involved testing work capability and providing preparatory work training adapted to the individual's challenges and skill level, in a sheltered setting with close follow-up. According to usual practice, participants were allocated to various sheltered businesses in the area providing different types of work settings, including food and catering, child care, mechanic services, transportation services, and warehouse handling, based on individual interests and goals as well as availability. The intervention was offered for up to two years, and the duration was customized to the individual's options on the labor market. The usual intensity of the intervention is full-time, with a requirement of $\geq 50 \%$ of full-time (15).

\section{Data collection}

Data were collected using questionnaires distributed at baseline, and 6 and 12 months after enrollment. For more information about data collection and management, see the study protocol (14). In order to increase the response rate to the primary outcome, non-respondents were contacted by telephone, text message, and e-mail. Participants who provided ambiguous responses to the primary outcome in the questionnaire were also contacted by telephone for clarification. In cases where contact was not obtained, log-books from the job specialists were used to provide information on the primary outcome for IPS participants ( $\mathrm{N}=7$ ).

\section{Outcomes}

Primary outcome: competitive employment (12-months follow-up). The primary outcome was any competitive employment during the 12-months follow-up. Competitive employment was defined as paid employment in the competitive labor market, and thus did not include subsidized or unpaid work. It was measured by self-report using a single item asking the participants to indicate the number of weeks, days, or hours worked in competitive employment during the first 12 months after enrollment. A dichotomous variable indicating any competitive work versus no competitive work was created.

Secondary employment-related outcomes (12-months and long-term follow-up). Additional standardized indicators of successful employment (16) during 12-months followup included percentage of participants ever working $\geq 20$ hours per week, total number of hours worked, weeks from enrollment to first job, and weeks worked at longestheld job, were also included in the questionnaires.

Register data on benefit recipiency and income from $\mathrm{NAV}$, and financial assistance and educational activity from Statistics Norway, will also be collected during long-term follow-up for up to five years after enrollment.

Secondary health-related outcomes (6- and 12-months follow-up). Secondary outcomes were level of disability, using the World Health Organization Disability Assessment Schedule (WHODAS) 2.0 (17); psychological distress, using the Hopkins Symptom Checklist (HSCL-25) $(18,19)$; severity of subjective health complaints, using the Subjective Health Complaints Inventory (SHC) (20); fatigue, using the Chalder Fatigue Questionnaire (CFQ) (21); coping, helplessness and hopelessness, using the Theoretically Originated Measure of the Cognitive Activation Theory of Stress (TOMCATS) (22); alcohol consumption, using the Alcohol Use Disorders Identification Test 
consumption questions (AUDIT-C) (23); and drug use, using the Drug Use Disorders Identification Test consumption questions (DUDIT-C) $(24,25)$. Global wellbeing was measured using a 10-point Cantril Ladder Scale (26) ranging from 1 (worst life possible) to 10 (best life possible) asking about the current situation, the situation one year ago, and one year in the future. This measure replaced the EQ-5D measuring quality of life described in the study protocol (14), in order to shorten the questionnaire. Higher scores on each scale indicated higher levels of the respective outcome. Measures of social support and illness perceptions, which were also included in the study protocol (14), will be investigated in a future paper examining moderators of treatment effects.

Fidelity and process measures for the IPS intervention. To assess the adherence to the evidence-based IPS Supported Employment Fidelity Scale (27), the external IPS trainer regularly conducted fidelity reviews throughout the project period. Each review was conducted over two consecutive days and involved document and calendar review, observations, and interviews of the different stakeholders. The scale consists of 25 items rated on a 5 -item behaviorally anchored scale with total scores ranging from 25-125; scores $\leq 73$ do not fulfill the minimal criteria for IPS.

IPS participants received additional questions at follow-up related to adherence to and satisfaction with the intervention. At 6- and 12-months follow-up, participants were asked to indicate on 5-point scales how satisfied they were with the intervention in general; how satisfied they were with their job specialist; and how useful it had been to participate in the intervention. In addition, IPS participants were asked at 6-months follow-up whether or not they had initiated at least one of the goals they had set with their job specialist during their first meetings (eg, finding references, drafting their $\mathrm{CV}$ ), and to indicate barriers and helpful factors for participation.

\section{Sample size}

A required sample size of 124 participants was estimated based on input data from previous IPS studies with a mean competitive employment rate of $61 \%$ for IPS and $23 \%$ for control groups (28). Calculations were performed using the Hmisc library in the statistical package $\mathrm{R}(29)$, based on a $5 \%$ significance level and a power of $90 \%$, accounting for stratified analyses to investigate treatment effects for two sub-groups (eg, gender).

\section{Statistical analysis}

Descriptive statistics on demographic and health-related characteristics were calculated for the total sample and each intervention group at baseline. Baseline differences between the groups, and between respondents and non-respondents at follow-up, were analyzed using chi square tests for dichotomous variables and independent t-tests for continuous variables.

Analysis on the primary outcome was conducted using chi square test comparing crude employment rates of participants in each group. The odds ratio (OR) was also calculated. Rates of working $\geq 20$ hours per week were compared with the same method, and number of hours worked were compared using t-tests. In order for effect sizes to be comparable across dichotomous and continuous outcomes, the effect size for differences between proportions was calculated using the arcsine formula (30).

For the secondary health-related outcomes, unadjusted differences between groups at each follow-up point were compared using t-tests. However, due to multiple observations for participants, at baseline and 6 and 12 months, and to adjust for missing observations and baseline ratings on each outcome, post hoc analyses with mixed effects regression models were also applied. In the mixed effect model, maximum likelihood estimation (MLE) will robustly adjust for missing observations. Using this approach accounts for complex structures of missing data (31).

All analyses followed the intention-to-treat (ITT) principle according to the randomized groups, regardless of compliance per protocol. The significance level was $\alpha=0.05$. Analyses were performed using IBM SPSS Statistics version 25.0 (IBM Corp, Armonk, NY, USA) and StataIC version 15 (StataCorp. College Station, TX, USA).

\section{Results}

\section{Participant flow}

A total of 98 participants were included and randomized (50 to IPS and 46 to TVR). Two participants were excluded before intervention and follow-up due to ineligibility according to the inclusion criteria (figure 1). The final sample consisted of 96 participants [68\% male, mean age 24 (SD 3.25) years].

The response rate for the primary outcome (main analysis) was $\mathrm{N}=83(86 \%)$, and there was no significant difference in response rate between the groups $(\mathrm{P}=0.098)$. The response rate on questionnaires with secondary outcomes was $72 \%$ at 6 -months follow-up, dropping to $64 \%$ at 12 -months follow-up. There was a significant difference in response rate between IPS and TVR groups at 6 -months follow-up $\left(\chi^{2}(1)=7.59\right.$, $\mathrm{P}=0.006$, Cohen's $d=0.57$ ) but no significant difference at 12 -months follow-up $(\mathrm{P}=0.602)$. 


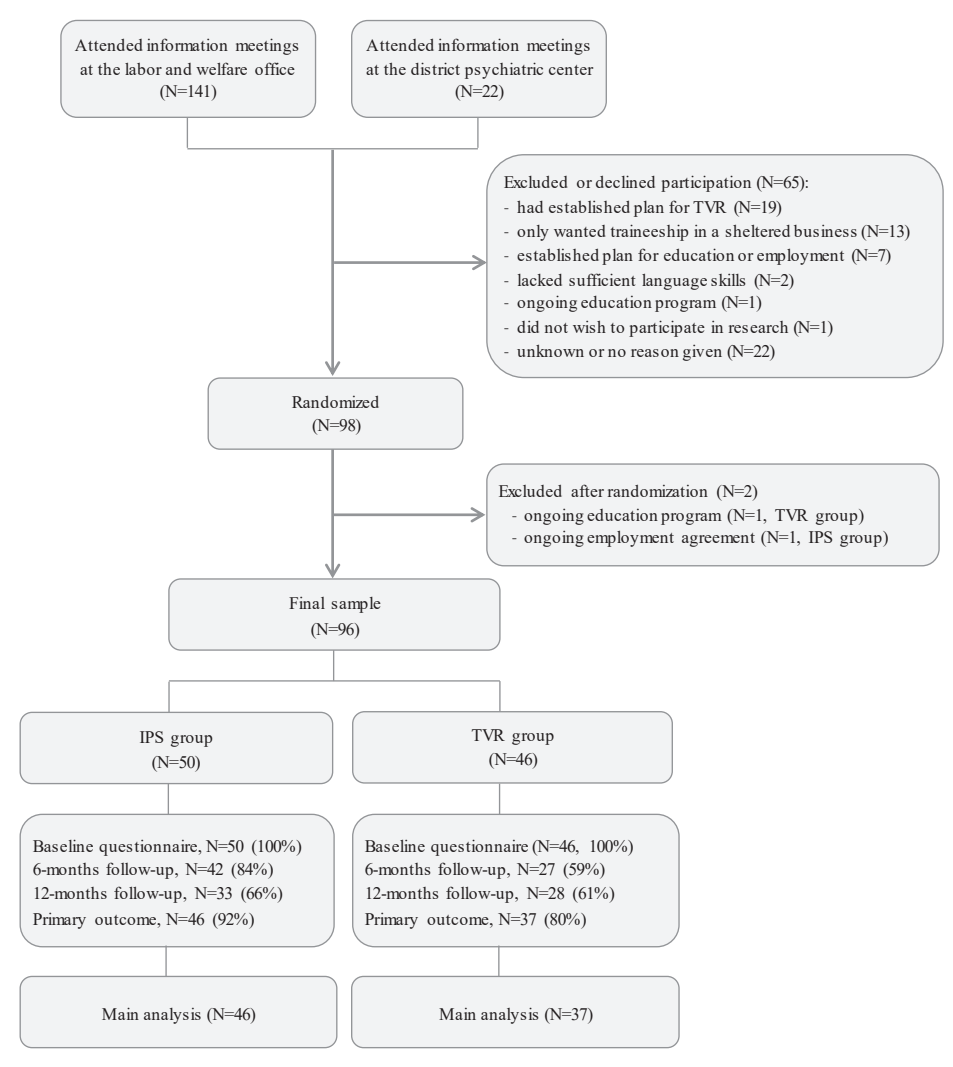

Figure 1. Participantflowthroughoutthetrial. [IPS=individual placementandsupport; TVR=traditional vocational rehabilitation.]

Table 1. Baseline demographic and health-related characteristics and comparison of groups. [IPS=individual placement and support; TVR=traditional vocational rehabilitation; $\mathrm{SD}=$ standard deviation; WHODAS=World Health Organization Disability Assessment Schedule; HSCL=Hopkins Symptom Checklist; $\mathrm{CFQ}=$ Chalder Fatigue Questionnaire; SHC=subjective health complaints; TOMCATS=theoretically originated measure of the cognitive activation theory of stress; AUDIT=Alcohol Use Disorders Identification Test; DUDIT=Drug Use Disorders Identification Test.]

\begin{tabular}{|c|c|c|c|c|c|c|c|c|c|}
\hline \multirow[t]{2}{*}{ Variables } & \multicolumn{4}{|c|}{ IPS (N=50) } & \multicolumn{4}{|c|}{ TVR (N=46) } & \multirow[b]{2}{*}{ P-value } \\
\hline & $\mathrm{N}$ & $\%$ & Mean & SD & $\mathrm{N}$ & $\%$ & Mean & SD & \\
\hline Age at inclusion & & & 23.96 & 3.46 & & & 23.85 & 3.04 & 0.867 \\
\hline Gender (male) & 32 & 64.00 & & & 33 & 71.74 & & & 0.418 \\
\hline Education (less than high-school) & 24 & 48.00 & & & 14 & 30.43 & & & 0.079 \\
\hline Previously held a job & 27 & 54.00 & & & 29 & 64.44 & & & 0.301 \\
\hline Previously attended employment scheme & 31 & 63.27 & & & 28 & 65.12 & & & 0.853 \\
\hline \multicolumn{10}{|l|}{ Reasons for unemployment ${ }^{a}$} \\
\hline Psychological problems & 25 & 50.00 & & & 26 & 56.52 & & & 0.522 \\
\hline Other health problems & 18 & 36.00 & & & 15 & 32.61 & & & 0.727 \\
\hline Non-health related problems & 18 & 36.00 & & & 14 & 30.43 & & & 0.563 \\
\hline WHODAS (0-48) & & & 8.18 & 8.12 & & & 9.07 & 6.92 & 0.566 \\
\hline $\mathrm{HSCL}(1-4)$ & & & 1.81 & 0.58 & & & 1.89 & 0.52 & 0.468 \\
\hline HSCL depression (1-4) & & & 1.91 & 0.69 & & & 2.00 & 0.59 & 0.464 \\
\hline HSCL anxiety (1-4) & & & 1.67 & 0.47 & & & 1.73 & 0.52 & 0.525 \\
\hline $\mathrm{CFQ}(0-33)$ & & & 12.93 & 5.60 & & & 13.96 & 6.07 & 0.394 \\
\hline CFQ physical (0-21) & & & 8.40 & 4.19 & & & 8.98 & 4.65 & 0.524 \\
\hline $\mathrm{CFQ}$ mental $(0-12)$ & & & 4.53 & 2.20 & & & 4.98 & 2.25 & 0.329 \\
\hline $\mathrm{SHC}(0-87)$ & & & 13.96 & 9.71 & & & 15.23 & 9.41 & 0.522 \\
\hline SHC musculoskeletal (0-24) & & & 3.88 & 4.02 & & & 4.46 & 3.89 & 0.480 \\
\hline SHC pseudoneurology (0-21) & & & 5.45 & 3.56 & & & 6.11 & 4.32 & 0.412 \\
\hline SHC gastrointestinal (0-21) & & & 2.64 & 3.33 & & & 2.12 & 2.27 & 0.375 \\
\hline Global well-being, current (1-10) & & & 4.69 & 1.58 & & & 5.03 & 2.00 & 0.363 \\
\hline Global well-being, past $(1-10)$ & & & 4.15 & 2.17 & & & 3.83 & 2.31 & 0.487 \\
\hline Global well-being, future (1-10) & & & 6.83 & 2.18 & & & 7.23 & 2.08 & 0.378 \\
\hline TOMCATS coping (1-4) & & & 2.63 & 0.76 & & & 2.62 & 0.68 & 0.944 \\
\hline TOMCATS helplessness (1-4) & & & 2.36 & 0.66 & & & 2.31 & 0.73 & 0.730 \\
\hline TOMCATS hopelessness (1-4) & & & 2.33 & 0.79 & & & 2.23 & 0.67 & 0.490 \\
\hline AUDIT-C (0-12) & & & 3.30 & 2.40 & & & 3.39 & 2.56 & 0.857 \\
\hline DUDIT-C (0-16) & & & 0.47 & 1.46 & & & 0.37 & 0.97 & 0.698 \\
\hline
\end{tabular}

a Participants could choose more than one option. 


\section{Baseline characteristics}

There were no significant differences between the groups on demographic or health-related variables at baseline (table 1). For more information on baseline characteristics, see Sveinsdottir et al. (32).

There were no significant baseline differences between respondents and non-respondents at 6-months follow-up. Respondents at 12-months follow-up were, however, more likely than non-respondents to be female $\left(\chi^{2}(1)=5.78, \mathrm{P}=0.016\right.$, Cohen's $\left.d=0.54\right)$ and to have more than a high school education $\left(\chi^{2}(1)=7.10, \mathrm{P}=0.008\right.$, Cohen's $d=0.55$ ), but did not differ significantly on health-related variables.

When comparing baseline differences between the reduced samples of respondents in the IPS and TVR groups, respondents to 6-months follow-up in the TVR group reported higher baseline global well-being (mean $5.73, \mathrm{SD}=1.96, \mathrm{~N}=22$ ) than respondents in the IPS group (mean 4.71, $\mathrm{SD}=1.69, \mathrm{~N}=35$ ), $t(55)=2.07, \mathrm{P}=0.043$, Cohen's $d=0.55$. There were no significant baseline differences between respondents to 12-months follow-up in the IPS and TVR groups.

\section{Primary and secondary employment-related outcomes}

Compared to the TVR group, a significantly higher proportion of the IPS group obtained competitive employment at any time during the 12-months follow-up period (table 2). A significantly higher proportion of the IPS group had also ever worked $\geq 20$ hours per week, and participants in the IPS group worked more hours on average, compared to the TVR group (table 2).

Due to problems in the data collection of weeks from enrollment to first job, and weeks worked at longest-held job, data on these outcomes were insufficient and are not reported.

The register data with long-term follow-up have a time lag, and will be reported after they are available.

\section{Secondary health-related outcomes}

Unadjusted analyses. Groups did generally not differ in secondary outcomes in the unadjusted analyses, with some exceptions in favor of the IPS group in severity of subjective health complaints and helplessness at 6-months follow-up, and in hopelessness at 12-months follow-up (table 3).

Adjusted post hoc analyses. In the adjusted post hoc analyses, participants in the IPS group reported significantly more positive effects on secondary outcomes of anxiety $(\mathrm{P}=0.045)$, subjective health complaints $(\mathrm{P}=0.001)$, pseudoneurology $(\mathrm{P}=0.033)$, helplessness $(\mathrm{P}=0.002)$, hopelessness $(\mathrm{P}=0.029)$, and drug use $(\mathrm{P}=0.043)$ compared to the TVR group at 6-months follow-up. With the exception of anxiety and pseudoneurology, effects were maintained at 12 months, for subjective health complaints $(\mathrm{P}=0.017)$, helplessness $(\mathrm{P}=0.017)$, hopelessness $(\mathrm{P}=0.006)$, and drug use $(\mathrm{P}=0.036)$. Participants in the IPS group also showed significantly lower levels of disability $(\mathrm{P}=0.038)$ and more optimism about future well-being $(\mathrm{P}=0.038)$ at 12 months compared to the TVR group.

Fidelity to the IPS model and participants' experiences with the intervention. Five fidelity reviews were conducted in June and December of 2015 and 2016 and in July 2017, coinciding with the time period of recruitment and 12-months follow-up. Due to the adaptions made to the IPS intervention noted above, fidelity items concerning integration with mental health treatment were rated as 1 (lowest score). The total score for the first review started at a low point of 47 , and there was a general increase up to a score of 77 at the last review. Three of five reviews were above the cut-off for "fair" fidelity (29).

At 6-months follow-up, most participants in the IPS group $(\mathrm{N}=36,95 \%)$ reported having initiated at least one of the goals they had set with their job specialist. At 6-months follow-up, $\mathrm{N}=30$ (77\%) were quite or very

Table 2. Primary and secondary employment-related outcomes, and comparison of groups. [SD=standard deviation; $\mathrm{OR}=$ odds ratio; $\mathrm{Cl}=$ confidence interval; IPS=individual placement and support; TVR=traditional vocational rehabilitation.]

\begin{tabular}{|c|c|c|c|c|c|c|c|c|c|}
\hline Variables & Group & $\mathrm{N}$ & $\%$ & Mean & SD & P-value & Cohen's d & OR & $95 \% \mathrm{Cl}$ \\
\hline \multicolumn{10}{|l|}{ Primary outcome } \\
\hline Competitively employed ${ }^{a}$ & $\begin{array}{l}\text { IPS } \\
\text { TVR }\end{array}$ & $\begin{array}{r}22 \\
3\end{array}$ & $\begin{array}{r}47.83 \\
8.11\end{array}$ & & & $<0.001$ & 0.96 & 10.39 & $2.79-38.68$ \\
\hline \multicolumn{10}{|l|}{ Secondary outcome } \\
\hline Ever worked $\geq 20$ hours per week ${ }^{b}$ & $\begin{array}{l}\text { IPS } \\
\text { TVR }\end{array}$ & $\begin{array}{r}14 \\
2\end{array}$ & $\begin{array}{r}33.33 \\
5.41\end{array}$ & & & 0.002 & 0.77 & 8.75 & $1.83-41.75$ \\
\hline Hours worked ${ }^{c}$ & $\begin{array}{l}\text { IPS } \\
\text { TVR }\end{array}$ & & & $\begin{array}{r}140.02 \\
13.95\end{array}$ & $\begin{array}{r}249.36 \\
55.48\end{array}$ & 0.002 & 0.70 & & \\
\hline
\end{tabular}

${ }^{\text {a }}$ Data for competitive employment were available for $\mathrm{N}=83$ ( $86 \%$ of the total sample, IPS N=46, $92 \%$ and TVR $\mathrm{N}=37,80 \%$ ).

${ }^{b}$ Data for working $\geq 20$ hours per week were available for $\mathrm{N}=79$ ( $82 \%$ of the total sample, IPS $\mathrm{N}=42,84 \%$ and TVR $\mathrm{N}=37,80 \%$ ).

c Data for hours worked were available for $\mathrm{N}=80$ ( $83 \%$ of the total sample, IPS group $\mathrm{N}=43,86 \%$ and TVR group $\mathrm{N}=37,80 \%$ ). 
satisfied with their job specialist, $\mathrm{N}=28(72 \%)$ were quite or very satisfied with the intervention, and $\mathrm{N}=26$ $(68 \%)$ reported that participation had been quite or very useful. At 12-months follow-up, $\mathrm{N}=20(63 \%)$ were quite or very satisfied with their job specialist, $\mathrm{N}=19(59 \%)$ were quite or very satisfied with the intervention, and $\mathrm{N}=16(50 \%)$ reported that participation had been quite or very useful. Challenges with illness was the most common barrier for participating in the intervention $(\mathrm{N}=18,53 \%)$ followed by the content of the intervention not meeting expectations $(\mathrm{N}=7,21 \%)$ and transportation challenges (eg, meeting the job specialist or potential employers) ( $\mathrm{N}=7,21 \%)$. The most commonly reported helpful factors were the availability of the job specialist $(\mathrm{N}=30,83 \%)$, having the choice of whether or not to disclose their illness to employers or others $(\mathrm{N}=29$, $83 \%)$, regular follow-up from the job specialist $(\mathrm{N}=25$, $81 \%$ ), and having specific steps in the individualized job search plan during the process $(\mathrm{N}=25,76 \%)$.

\section{Discussion}

The SEED trial compared two contrasting vocational rehabilitation approaches for young adults assessed as having impaired work capability and who were not in education, employment, or training. IPS was superior to TVR in increasing participation in competitive employment among this group.
Participants who received IPS were more likely to be competitively employed during one year follow-up compared to participants receiving treatment as usual in TVR. Additionally, IPS participants worked more hours, and more often worked $\geq 20$ hours per week. These results, together with the fidelity and process measures, show that the IPS methodology can be applied to young adults at risk of early disability due to social or healthrelated problems that do not necessarily involve mental illness. The findings are in line with previous literature of IPS for patients with severe mental illness, showing that IPS is more effective than control conditions in promoting competitive employment (33). The employment rates of $48 \%$ versus $8 \%$ are similar to findings from a recent Swedish IPS study (34).

Only $8 \%$ of TVR participants obtained any competitive employment during follow-up. Although the TVR intervention involves prevocational training with close follow-up aimed at finding a job (15), the sheltered nature of the approach may preclude competitive employment by placing participants in a training situation outside the labor market and in an environment with others who are in the same situation. Although the current study does not have data on the duration and intensity of the intervention for the individual participants, the results indicate that participation in TVR may have left little time for efforts aimed toward attaining competitive employment. The results strongly suggest that providing direct support to finding and keeping competitive jobs rather than preparing clients in sheltered training settings is a more

Table 3. Secondary health-related outcomes for each group at 6- and 12-months follow-up, and comparison of groups (unadjusted analyses). [IPS=individual placement and support; TVR=traditional vocational rehabilitation; WHODAS=World Health Organization Disability Assessment Schedule; HSCL=Hopkins Symptom Checklist; CFQ=Chalder Fatigue Questionnaire; SHC=subjective health complaints; TOMCATS=theoretically originated measure of the cognitive activation theory of stress; AUDIT=Alcohol Use Disorders Identification Test; DUDIT=Drug Use Disorders Identification Test.]

\begin{tabular}{|c|c|c|c|c|c|c|c|c|c|c|c|c|c|c|c|c|}
\hline & \multicolumn{8}{|c|}{ 6-months follow-up } & \multicolumn{8}{|c|}{ 12-months follow-up } \\
\hline & \multicolumn{3}{|c|}{ IPS } & \multicolumn{3}{|c|}{ TVR } & \multirow[b]{2}{*}{$\mathrm{P}$-value } & \multirow[b]{2}{*}{ Cohen's d } & \multicolumn{3}{|c|}{ IPS } & \multicolumn{3}{|c|}{ TVR } & \multirow[b]{2}{*}{ P-value } & \multirow[b]{2}{*}{ Cohen's d } \\
\hline & $\mathrm{N}$ & Mean & SD & $\mathrm{N}$ & Mean & SD & & & $\mathrm{N}$ & Mean & SD & $\mathrm{N}$ & Mean & SD & & \\
\hline WHODAS (0-48) & 37 & 10.37 & 9.47 & 24 & 9.83 & 8.64 & 0.820 & 0.06 & 31 & 9.70 & 7.31 & 25 & 13.30 & 8.19 & 0.088 & 0.46 \\
\hline HSCL total (1-4) & 38 & 1.74 & 0.59 & 24 & 1.95 & 0.54 & 0.165 & 0.37 & 30 & 1.79 & 0.63 & 26 & 2.00 & 0.60 & 0.220 & 0.33 \\
\hline HSCL depression (1-4) & 38 & 1.84 & 0.72 & 24 & 2.04 & 0.56 & 0.254 & 0.31 & 30 & 1.89 & 0.74 & 26 & 2.15 & 0.72 & 0.183 & 0.36 \\
\hline HSCL anxiety (1-4) & 38 & 1.60 & 0.49 & 24 & 1.82 & 0.60 & 0.115 & 0.41 & 30 & 1.65 & 0.53 & 26 & 1.77 & 0.55 & 0.403 & 0.23 \\
\hline CFQ total (0-33) & 38 & 14.05 & 6.34 & 24 & 14.73 & 4.74 & 0.656 & 0.12 & 30 & 14.19 & 5.99 & 26 & 14.52 & 5.07 & 0.823 & 0.06 \\
\hline CFQ physical (0-21) & 38 & 9.32 & 4.77 & 24 & 9.86 & 3.50 & 0.631 & 0.13 & 30 & 9.55 & 4.30 & 26 & 9.42 & 3.80 & 0.903 & 0.03 \\
\hline CFQ mental (0-12) & 38 & 4.74 & 2.37 & 24 & 4.88 & 1.88 & 0.810 & 0.06 & 30 & 4.63 & 2.40 & 26 & 5.08 & 2.00 & 0.460 & 0.20 \\
\hline SHC total $(0-87)$ & 36 & 12.44 & 10.15 & 24 & 18.13 & 11.58 & 0.049 & 0.52 & 27 & 14.01 & 10.35 & 25 & 18.05 & 10.63 & 0.172 & 0.38 \\
\hline SHC musculoskeletal (0-24) & 36 & 3.47 & 3.68 & 24 & 4.99 & 4.73 & 0.167 & 0.36 & 28 & 4.00 & 4.11 & 26 & 5.60 & 3.89 & 0.149 & 0.40 \\
\hline SHC pseudoneurology (0-21) & 37 & 5.32 & 4.86 & 24 & 7.83 & 4.87 & 0.055 & 0.51 & 28 & 5.84 & 4.19 & 25 & 6.85 & 4.49 & 0.397 & 0.23 \\
\hline SHC gastrointestinal (0-21) & 37 & 2.61 & 3.56 & 24 & 2.65 & 2.46 & 0.958 & 0.01 & 27 & 2.85 & 3.44 & 25 & 2.67 & 3.18 & 0.846 & 0.05 \\
\hline Global well-being, current (1-10) & 36 & 4.56 & 1.73 & 22 & 5.14 & 2.49 & 0.344 & 0.27 & 30 & 4.83 & 1.90 & 25 & 4.72 & 2.13 & 0.836 & 0.06 \\
\hline Global well-being, past (1-10) & 36 & 3.36 & 1.74 & 22 & 3.91 & 1.97 & 0.274 & 0.29 & 31 & 3.65 & 1.76 & 25 & 4.20 & 2.35 & 0.317 & 0.26 \\
\hline Global well-being, future (1-10) & 35 & 6.36 & 2.58 & 22 & 6.77 & 2.56 & 0.555 & 0.16 & 30 & 8.53 & 10.87 & 25 & 6.06 & 2.61 & 0.272 & 0.31 \\
\hline TOMCATS coping (1-4) & 39 & 2.77 & 0.78 & 23 & 2.83 & 0.49 & 0.725 & 0.09 & 29 & 2.79 & 0.62 & 25 & 2.76 & 0.88 & 0.872 & 0.04 \\
\hline TOMCATS helplessness (1-4) & 39 & 2.34 & 0.77 & 24 & 2.79 & 0.69 & 0.021 & 0.62 & 29 & 2.40 & 0.71 & 26 & 2.67 & 0.69 & 0.167 & 0.38 \\
\hline TOMCATS hopelessness (1-4) & 37 & 2.18 & 0.66 & 24 & 2.45 & 0.67 & 0.124 & 0.41 & 29 & 2.08 & 0.75 & 25 & 2.52 & 0.83 & 0.046 & 0.55 \\
\hline AUDIT-C (0-12) & 38 & 3.08 & 2.55 & 26 & 3.62 & 3.48 & 0.479 & 0.18 & 31 & 2.81 & 2.40 & 26 & 3.58 & 2.93 & 0.280 & 0.29 \\
\hline DUDIT-C (0-16) & 37 & 0.14 & 0.59 & 26 & 0.85 & 2.17 & 0.114 & 0.45 & 30 & 0.27 & 0.87 & 24 & 1.04 & 2.74 & 0.194 & 0.38 \\
\hline
\end{tabular}


effective way to reintegrate vulnerable NEET into the competitive labor market.

Although participants were generally satisfied with the IPS intervention, the program scored low on fidelity. The quality of the implementation was thus below fidelity benchmarks attained in many prior IPS studies. One continuing weakness in IPS implementation was the lack of integration between employment and health care services. Other issues were low scores on providing follow-up after employment, assertive engagement and outreach for clients who missed appointments, agency focus on competitive employment, and job specialists spending too much time on non-vocational services. Previous studies have shown that higher fidelity is associated with better employment outcomes $(35,36)$, which may also be true for this population, but this cannot be demonstrated until a high fidelity IPS program has been evaluated. These issues should therefore be considered in future efforts to provide IPS to non-psychiatric populations and may, for example, require the establishment of more structured routines to integrate any relevant health services in the intervention.

Participants mainly reported psychological problems as a reason for unemployment at baseline, which mirrors the statistics of youth in high-income countries (6) as well as young disability benefit recipients in Norway (37). Other health or non-health related reasons were however also common, which illustrates the sample's diversity in terms of type of social and health-related problems compared to previous IPS trials. Baseline characteristics among the participants are discussed in more detail in a previous paper (32), showing that the prevalence of adverse social experiences (ie, bullying and violence) was highly prevalent, while more than half of participants also reported scores above predefined cut-offs for psychological distress as well as alcohol use, and about one third reported severe disability. Findings on secondary health-related outcomes at follow-up were inconsistent, but indicated that IPS also had more favorable effects on some non-vocational outcomes. Unadjusted analyses showed few significant findings, but the loss to follow-up resulted in reduced power and possibly increased risk of type-II error. In the adjusted analyses, participants in the IPS group had significantly less disability, subjective health complaints, drug use, helplessness and hopelessness, and a more optimistic view on future well-being, compared to the TVR group. The findings provide an interesting addition to the existing IPS literature, which has generally not found effects on non-vocational outcomes (9). The finding that participants felt more helpless and hopeless after receiving TVR compared to IPS, indicates that traditional sheltered interventions may preclude individual's feelings of control and promote the belief that the actions they take may not have any effect on their situation, or even make it worse (38). The current study is also the first to demonstrate positive outcomes of IPS on drug use and optimism about future well-being. Findings on disability and subjective health complaints are however in line with a recent Norwegian trial of patients with moderate and severe mental illness (39) but appear weaker and more inconsistent on outcomes related to mental health. This may be partly explained by characteristics of the sample in the current study, who generally had less severe psychiatric symptoms than IPS participants in previous trials. It is however also important to note that participation in IPS was not associated with any negative impact on health, even though participants represented a vulnerable group qualified for sheltered work training. This aligns with the previous research on IPS (40) and suggests that concerns for potential detrimental health effects of non-sheltered approaches for this group may be unsubstantiated.

\section{Strengths and limitations}

The main strengths of the current study include the rigorous RCT design and the investigation of IPS for a new and important target group. The study addresses an established need in the literature for effective interventions to help NEET enter the labor market (1) and indicates that IPS may have the potential to forestall entry into the disability system for this group.

The study also has several limitations. The relatively small sample size precluded subgroup analyzes and may reduce generalizability. Although findings on the main outcome were strong, the confidence interval was large, indicating that there is need for larger replication studies to confirm the results. The power calculation was only performed for the primary outcome, and the small sample size reduces the chance of reaching statistical significance, in particular for secondary outcomes with missing data at follow-up. Differential attrition at 6-months is a threat to internal validity. This was mitigated by applying mixed effects models (with MLE), which is a recommended approach to handle complex structures of missing data (31). Due to the considerable number of secondary outcome measures included in the study, alpha inflation is a concern, suggesting caution in interpreting these findings.

The dichotomous primary outcome is a simplistic measure, which may be too crude to capture the many aspects of employment (16). It is however the most commonly used outcome in previous IPS studies and serves as a useful general-purpose measure (16), which was supplemented with data on ever working $\geq 20$ hours per week and the continuous measure of hours worked. Due to problems in the data collection we were however unable to collect sufficient data on further indicators of successful employment, which represents a limitation 
to the study. The use of self-report data for competitive employment may also increase the risk of bias, and it is also uncertain whether the effects will hold up in the long-term. This will be followed up in a subsequent paper with objective register data from the NAV, which will provide a more conservative, yet reliable and complete measure of labor market participation for up to five years after enrollment. These data will also form the basis for a cost-benefit analysis.

Data on potential harms was not collected in the study. There were routines for reporting and handling any harms/adverse effects reported directly to the project group or to the job specialists, but there were no such reports.

\section{Concluding remarks}

The results showed that IPS can be successfully applied to NEET with impaired work capability due to various social or health-related problems. IPS was superior to TVR in increasing participation in competitive employment among this group, and also promoted improvements in level of disability, subjective health, feelings of helplessness and hopelessness, and drug use, when adjusted for missing observations. Based on the results from the current study, we recommend that IPS services should be offered to improve labor market participation among young adults at risk of early work disability.

\section{Acknowledgements}

We would like to thank all the participants who took part in the project, and the staff at Fretex Western Norway, NAV, and IPS Norge for their collaboration. We also thank Line Solberg, Adrian Løken, and Nina Konglevoll for their efforts as research assistants. The Research Council of Norway funded the SEED trial (project \#227002/H20) but did not have any role in the study design, data collection or analysis, or decisions to publish. The authors declare no conflicts of interest.

\section{References}

1. Eurofound. NEETs - Young people not in employment, education or training: Characteristics, costs and policy responses in Europe. Luxembourg: Publications Office of the European Union; 2012.

2. Eurofound. Exploring the diversity of NEETs. Luxembourg: Publications Office of the European Union; 2016.

3. Paul KI, Moser K. Unemployment impairs mental health: Meta-analyses. J Vocat Behav. 2009;74(3):264-82. https://doi. org/10.1016/j.jvb.2009.01.001

4. McKee-Ryan F, Song Z, Wanberg CR, Kinicki AJ. Psychological and physical well-being during unemployment: a meta-analytic study. J Appl Psychol. 2005;90(1):53-76. https://doi.org/10.1037/0021-9010.90.1.53

5. Norwegian Labor and Welfare Administration. Utviklingen i uføretrygd per 30. september 2018 [The development of disability benefits, by September 30th 2018]. Oslo: NAV; 2018.

6. Gore FM, Bloem PJN, Patton GC, Ferguson J, Joseph V, Coffey $\mathrm{C}$, et al. Global burden of disease in young people aged 10-24 years: a systematic analysis. Lancet. 2011;377(9783):2093102.https://doi.org/10.1016/S0140-6736(11)60512-6

7. Mawn L, Oliver EJ, Akhter N, Bambra CL, Torgerson C, Bridle $\mathrm{C}$, et al. Are we failing young people not in employment, education or training (NEETs)? A systematic review and metaanalysis of re-engagement interventions. Systematic reviews. 2017;6(1):16. https://doi.org/10.1186/s13643-016-0394-2

8. Drake RE, Bond GR, Goldman HH, Hogan MF, Karakus M. Individual Placement And Support Services Boost Employment For People With Serious Mental Illnesses, But Funding Is Lacking. Health affairs (Project Hope). 2016;35(6):1098-105. https://doi.org/10.1377/hlthaff.2016.0001

9. Kukla M, Bond GR. A randomized controlled trial of evidencebased supported employment: Nonvocational outcomes. J Vocat Rehabil. 2013;38:91-8.

10. Luciano A, Bond GR, Drake RE. Does employment alter the course and outcome of schizophrenia and other severe mental illnesses? A systematic review of longitudinal research. Schizophrenia research. 2014;159(2-3):312-21. https://doi. org/10.1016/j.schres.2014.09.010

11. Drake RE, Bond GR, Becker DR. Individual Placement and Support: An Evidence-Based Approach to Supported Employment. New York: Oxford University Press, USA; 2012. https://doi.org/10.1093/acprof:oso/9780199734016.001.0001

12. Bond GR, Drake RE, Pogue JA. Expanding Individual Placement and Support to Populations With Conditions and Disorders Other Than Serious Mental Illness. Psychiatric services (Washington, DC). 2019. https://doi.org/10.1176/ appi.ps.201800464

13. LOV-2008-06-20-44 §4a. The Act on Medical and Health Research (the Health Research Act). http://app.uio.no/ub/ ujur/oversatte-lover/data/lov-20080620-044-eng.pdf.

14. Sveinsdottir V, Tveito TH, Bond GR, Grasdal AL, Lie SA, Reme SE. Protocol for the SEED-trial: Supported Employment and preventing Early Disability. BMC Public Health. 2016;16:579.https://doi.org/10.1186/s12889-016-3280-x

15. Norwegian Labor and Welfare Administration. Kravspesifikasjon for tiltak i skjermede virksomheter [Requirement Specification for Services in Sheltered Businesses]. Oslo: NAV; 2011 [cited 2017 May 5th]. Available from: https://www.nav.no/_attachment/87667.

16. Bond GR, Campbell K, Drake RE. Standardizing measures in four domains of employment outcomes for individual placement and support. Psychiatric services (Washington, DC). 2012;63(8):751-7. https://doi.org/10.1176/appi. 
ps. 201100270

17. Üstün TB, Kostanjsek N, Chatterji S, Rehm J. Measuring health and disability: manual for WHO Disability Assessment Schedule (WHODAS 2.0). Geneva: World Health Organization; 2010.

18. Derogatis LR, Lipman RS, Rickels K, Uhlenhuth EH, Covi L. The Hopkins Symptom Checklist (HSCL): a selfreport symptom inventory. Behav Sci. 1974;19(1):1-15. https://doi.org/10.1002/bs.3830190102

19. Winokur A, Winokur DF, Rickels K, Cox DS. Symptoms of emotional distress in a family planning service: stability over a four-week period. Br J Psychiatry. 1984;144:395-9. https://doi.org/10.1192/bjp.144.4.395

20. Eriksen HR, Ihlebaek C, Ursin H. A scoring system for subjective health complaints (SHC). Scandinavian journal of public health. 1999;27(1):63-72. https://doi.org/10.1177/140 34948990270010401

21. Chalder T, Berelowitz G, Pawlikowska T, Watts L, Wessely $\mathrm{S}$, Wright D, et al. Development of a fatigue scale. Journal of psychosomatic research. 1993;37(2):147-53. https://doi. org/10.1016/0022-3999(93)90081-P

22. Odeen M, Westerlund H, Theorell T, Leineweber C, Eriksen HR, Ursin H. Expectancies, Socioeconomic Status, and SelfRated Health: Use of the Simplified TOMCATS Questionnaire. International journal of behavioral medicine. 2013;20(2):24251.https://doi.org/10.1007/s12529-012-9221-x

23. Bush K, Kivlahan DR, McDonell MB, Fihn SD, Bradley KA. The AUDIT alcohol consumption questions (AUDIT-C): an effective brief screening test for problem drinking. Ambulatory Care Quality Improvement Project (ACQUIP). Alcohol Use Disorders Identification Test. Arch Intern Med. 1998;158(16):1789-95. https://doi.org/10.1001/ archinte.158.16.1789

24. Berman AH, Bergman H, Palmstierna T, Schlyter F. DUDIT (Drug Use Disorders Identification Test) Manual. Stockholm: Karolinska Institutet; 2003. https://doi.org/10.1037/t02890000

25. Sinadinovic K, Wennberg P, Berman AH. Targeting problematic users of illicit drugs with Internet-based screening and brief intervention: a randomized controlled trial. Drug Alc Dep. 2012;126(1-2):42-50.https://doi.org/10.1016/j. drugalcdep.2012.04.016

26. Cantril H. The pattern of human concerns. New Brunswick, NJ: Rutgers University Press; 1965.

27. Becker DR, Swanson S, Reese SL, Bond GR, McLeman BM. Supported Employment Fidelity Review Manual 3ed. Lebanon, NH: Dartmouth Psychiatric Research Center; 2015.
28. Bond GR, Drake RE, Becker DR. An update on randomized controlled trials of evidence-based supported employment. Psychiatr Rehabil J. 2008;31(4):280-90. https://doi. org/10.2975/31.4.2008.280.290

29. R Core Team. R: A language and environment for statistical computing. Vienna, Austria: R Foundation for Statistical Computing; 2013.

30. Lipsey MW. Design Sensitivity: Statistical Power for Experimental Research. Newbury Park, CA: SAGE; 1990.

31. Rabe-Hesketh S, Skrondal A. Multilevel and Longitudinal Modeling Using Stata: Continuous Responses, Third Edition. College Station, TX: Stata Press; 2012.

32. Sveinsdottir V, Eriksen HR, Baste V, Hetland J, Reme SE. Young Adults at Risk of Early Work Disability: Who are they? BMC Public Health. 2018;18:1176.https://doi.org/10.1186/ s12889-018-6095-0

33. Modini M, Tan L, Brinchmann B, Wang MJ, Killackey E, Glozier N, et al. Supported employment for people with severe mental illness: systematic review and meta-analysis of the international evidence. Bri J Psych. 2016;209(1):14-22. https://doi.org/10.1192/bjp.bp.115.165092

34. Bejerholm U, Areberg C, Hofgren C, Sandlund M, Rinaldi M. Individual Placement and Support in Sweden-A randomized controlled trial. Nord J Psychiatry. 2014;1:1-10. https://doi.org/10.3109/08039488.2014.929739

35. Bond GR, Becker DR, Drake RE. Measurement of Fidelity of Implementation of Evidence-Based Practices: Case Example of the IPS Fidelity Scale. Clin Psychol-Sci Pr. 2011;18(2):12641. https://doi.org/10.1111/j.1468-2850.2011.01244.x

36. Bond GR, Peterson AE, Becker DR, Drake RE. Validation of the Revised Individual Placement and Support Fidelity Scale (IPS-25). Psych Ser (Washington, DC). 2012;63(8):758-63. https://doi.org/10.1176/appi.ps.201100476

37. Norwegian Labor and Welfare Administration. Utviklingen i uførediagnoser per 30. juni 2015 [The development of disability benefit diagnoses, by June 30th 2015]. Oslo: NAV; 2018.

38. Ursin H, Eriksen HR. The cognitive activation theory of stress. Psychoneuroendocrinology. 2004;29(5):567-92. https://doi. org/10.1016/S0306-4530(03)00091-X

39. Reme SE, Monstad K, Fyhn T, Sveinsdottir V, Lovvik C, Lie SA, et al. A randomized controlled multicenter trial of individual placement and support for patients with moderateto-severe mental illness. Scand J Work Environ Health. 2019;45(1):33-41. https://doi.org/10.5271/sjweh.3753

40. Luciano A, Drake RE, Bond GR, Becker DR, Carpenter-Song E, Lord S, et al. Evidence-based supported employment for people with severe mental illness: Past, current, and future research. J Vocat Rehabil. 2014;40(1):1-13.

Received for publication: 27 December 2018 